\title{
The structure of the nuclear stellar cluster of the Milky Way
}

\author{
Rainer Schödel ${ }^{1}$ and Andreas Eckart ${ }^{1,2}$ \\ ${ }^{1}$ I. Physikalisches Institut, Universität zu Köln, Zülpicher Str. 77, 50937 Köln, Germany \\ ${ }^{2}$ Max-Planck-Institut für Radioastronomie, Auf dem Hügel 69, 53121 Bonn, Germany \\ email: rainer@ph1.uni-koeln.de, eckart@ph1.uni-koeln.de
}

\begin{abstract}
High-resolution adaptive optics observations of the inner $0.5 \mathrm{pc}$ of the Milky Way with multiple intermediate band filters are presented. From the images, stellar number counts and a detailed map of the interstellar extinction were extracted. The extinction map is consistent with a putative southwest-northeast aligned outflow from the central arcseconds.

An azimuthally averaged, crowding and extinction corrected stellar density profile presents clear evidence for the existence of a stellar cusp around Sgr A*. Several density peaks are found in the cluster that may indicate clumping of stars, possibly related to the last epoch of star formation in the Galactic Center. An analysis of stars in the brightness range $14.25<\operatorname{mag}_{\mathrm{K}}<$ 15.75 shows possible signs of mass segregation.
\end{abstract}

Keywords. Stellar dynamics - Galaxy: center - Galaxy: nucleus

\section{Introduction}

The center of our Milky Way is located at a distance of $7.6 \pm 0.3 \mathrm{kpc}$ and contains a $3.6 \pm 0.3 \times 106 \mathrm{M}_{\odot}$ black hole $(\mathrm{BH})$, Sagittarius $\mathrm{A}^{*}$ (Sgr A*) (Schödel, Ott, Genzel et al. 2003; Ghez, Duchêne, Matthews et al. 2003; Eisenhauer, Genzel, Alexander et al. 2005). It is the only nucleus of a normal spiral galaxy that can be resolved in detail by observations. When the age of a galaxy is older than the relaxation time due to twobody encounters, the formation of a density excess around the central black hole, the so-called stellar cusp, is expected. The density in this cusp decreases from the black hole with a power law. The index of this power-law ranges between $-1 / 2$ and $-7 / 4$, depending on the stellar population and formation history of the cusp (Bahcall \& Wolf 1976; Bahcall \& Wolf 1977; Lightman \& Shapiro 1977; Murphy, Cohn \& Durisen 1991). Since the Galactic Center (GC) can be resolved into individual stars by observations, it is the template object, where to look for a stellar cusp. The first clear evidence for a density excess in the central arcseconds around Sgr A* was only obtained after adaptive optics (AO) became available at 8m-class telescopes (Genzel, Schödel, Ott et al. 2003). We present new $\mathrm{AO}$ observations of the central $0.5 \mathrm{pc}$ of the GC. The images were used for a thorough analysis of the GC stellar cluster, determining not only the two-dimensional density structure of the cluster, but also taking into account interstellar extinction and analysing the distribution of stars in different brightness ranges. Here, we can only give a very brief introduction into the data, their analysis, and the results. The interested reader is referred to the article by Schödel et al. (2006, submitted to A\&A).

\section{Observations and data reduction}

AO imaging observations were acquired with NACO/VLT im June and July 2004, using intermediate band (IB) filters of $0.06 \mu \mathrm{m}$ width, centered at 2.00, 2.06, 2.24, 2.27, and $2.30 \mu \mathrm{m}$ (see left panel in Fig. 1). StarFinder (Diolaiti, Bendinelli, Bonaccini et al. 2000) was used for point source detection and photometry via PSF fitting. 

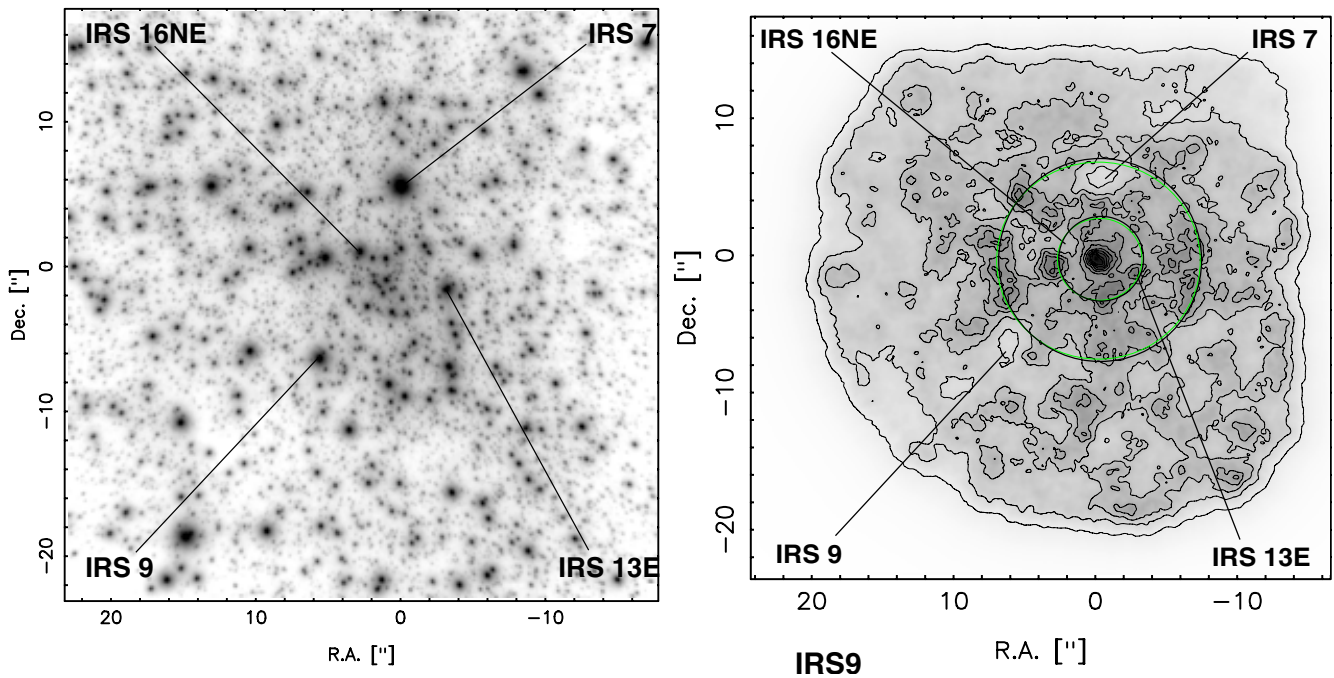

Figure 1. Left: Sum of the $2.27+2.30 \mu \mathrm{m}$ IB filter NACO images. This image was used for the star counts. Offsets from Sgr A* in right ascension and declination. North is up and east is to the left. Right: Adaptively smoothed, crowding and extinction corrected map of the stellar surface density. The smoothing was performed such that 40 stars contributed to the density at each point in the map. The smoothing radius varies between $0.6^{\prime \prime}$ near $\mathrm{Sgr} \mathrm{A}^{*}$ and $2^{\prime \prime}$ near the edge of the field. IRS 7, IRS 9 and IRS 16NE are particularly bright stars. The detection of faint stars is suppressed in their environment. The low density near these stars is therefore an artefact. The circles around Sgr A* indicate radial distances of $3^{\prime \prime}$ and $7^{\prime \prime}$. At these distances several density peaks appear to be concentrated. Note that bumps in the azimuthally averaged surface density can be seen at these distances in Fig. 3.

\section{Results}

Extinction. The IB 2.00 to 2.27 images were used to construct a map of interstellar extinction. The near-infrared (NIR) spectra of stars are largely free from strong emission or absorption features at these wavelengths and $A_{\mathrm{K}}$ can be determined from differential reddening. An extinction law $A_{\lambda} \propto \lambda^{-1.75}$ was assumed (Draine 1989). The median of all measurements within $2^{\prime \prime}$ of a given point in the FOV was taken. The resulting extinction map is shown in the left panel of Fig. 2. The $1 \sigma$ uncertainty of the map is $\leqslant 0.1 \mathrm{mag}$, except in the regions with highest extinction, where it is $\leqslant 0.2 \mathrm{mag}$ due to the reduced number of detected stars. It is intriguing to note that the so called mini-cavity can be distinguished in the extinction map. It has been suggested in previous work that the mini-cavity is caused by fast winds from the Helium stars in the central arcseconds or by an outflow from Sgr A* (Lutz, Krabbe \& Genzel 1993; Melia, Coker \& Yusef-Zadeh 1996; Yusef-Zadeh, Roberts \& Biretta 1998). The southwest-northeast aligned channellike feature that includes the mini-cavity in the extinction map is consistent with reduced extinction along a potential outflow along this direction (see Muzic et al., in prep.).

Stellar number density. The right panel of Fig. 1 shows the extinction corrected twodimensional stellar surface density in the central 0.5 pc of the GC. It can be seen that the GC stellar cluster appears fairly circularly symmetric. A strong density peak is visible centered on Sgr A*. Some regions of reduced density can be attributed to the presence of extremely bright stars, such as IRS 7, that hinder source detection in their immediate environment. It can be also seen that the stellar surface density does not decrease in a homogeneous way from the center, but that there are several density peaks present in the field, preferentially located at radial distances of $3^{\prime \prime}$ and $7^{\prime \prime}$ from Sgr A* (see circles 


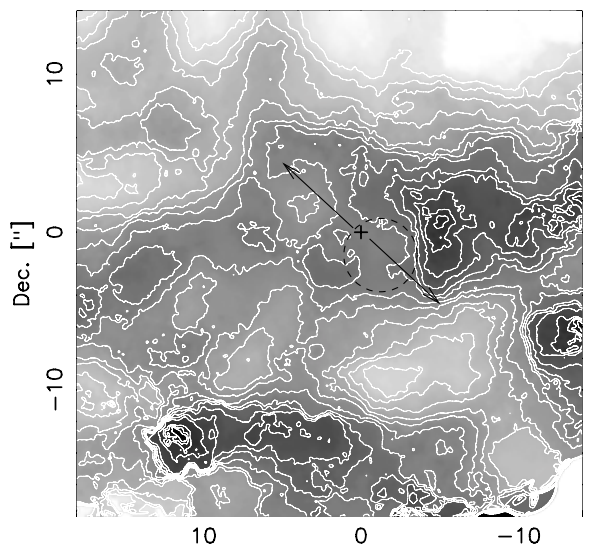

R.A. ["]

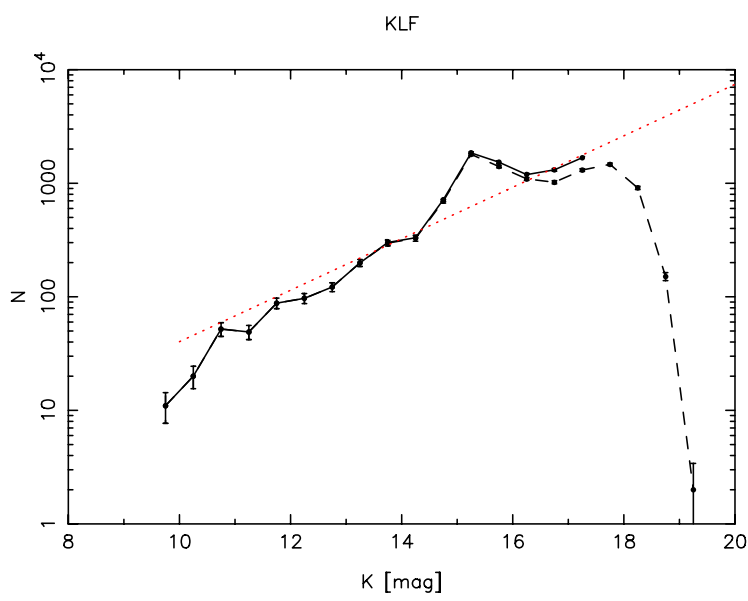

Figure 2. Left: Map of the interstellar extinction at $2.157 \mu \mathrm{m}$ derived from NACO intermediate band imaging at $2.00,2.06,2.24$, and $2.27 \mu \mathrm{m}$. The contours are spaced from $\mathrm{A}_{2.157 \mu \mathrm{m}}=2.0-3.5$ at intervals of 0.1. The cross indicates the position of Sgr A* and the dashed circle the approximate location of the mini-cavity. The arrows indicate a putative southwest-northeast aligned outflow from the central arcseconds. Right: KLF of the GC stellar cluster; dashed: raw counts, straight line: completeness corrected counts. The bump around $\operatorname{mag}_{\mathrm{K}}=15$ is due to horizontal branch/red clump (HB/RC) stars. The dotted line is a simple power law fit, omitting the HB/RC bump.
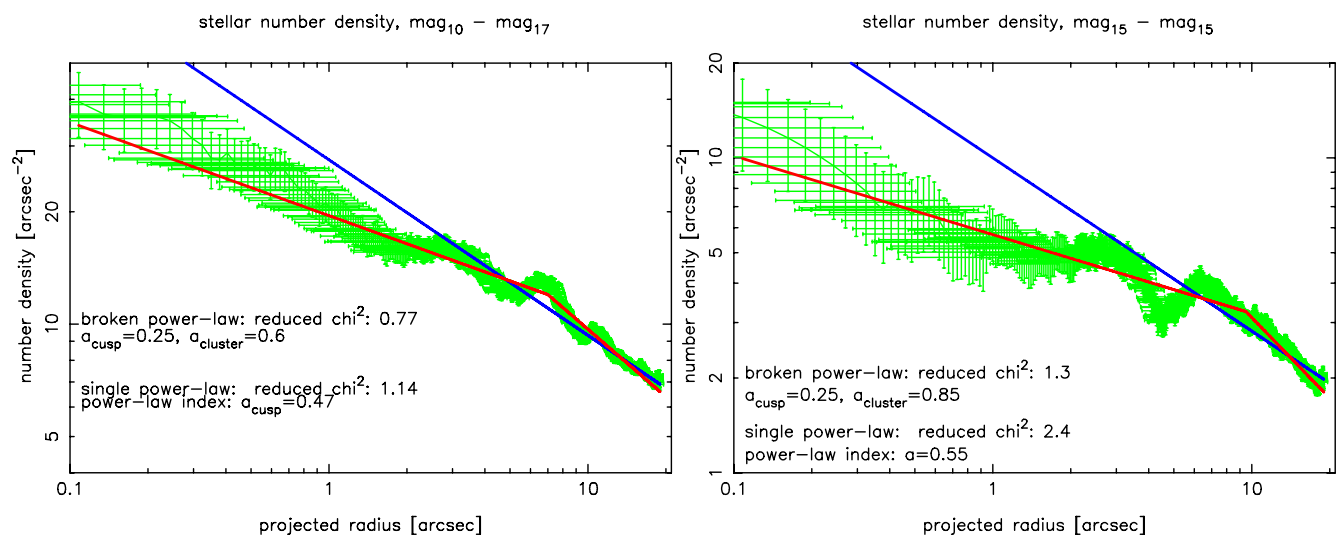

Figure 3. Left: Azimuthally averaged extinction and crowding corrected surface density vs. distance from Sgr A*. Fits to the data with a single (blue) and with a broken (red) power-law are indicated, along with the corresponding power-law indices and reduced $\chi^{2}$ values.

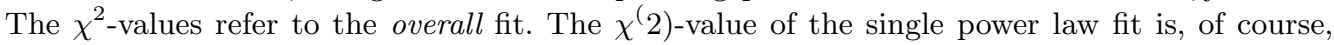
much worse in the central arcseconds. Right: The same as the left panel, but only for stars with $14.75<\operatorname{mag}_{\mathrm{K}}<15.75$.

in right panel of Fig. 1). One of these clumps is coincident with the dense co-moving group of stars in the IRS 13E complex (Maillard, Paumard, Stolovy et al. 2004; Schödel, Eckart, Iserlohe et al. 2005).

The azimuthally averaged extinction and crowding corrected surface density is presented in the left panel of Fig. 3. Power-laws were fitted to the plot of the stellar surface density. As can be seen in the Figure, a broken power law is necessary in order to provide a reasonable fit for the innermost arcseconds. The least-squares fit results in a broken 
power law with a break radius of $7 \pm 1^{\prime \prime}$, a power-law index of $\alpha=0.6 \pm 0.1$ for the outer part, and of $\alpha_{\text {cusp }}=0.25 \pm 0.1$ for the inner part of the cluster.

Evidence for mass segregation. The K-band luminosity function (KLF) of the cluster is shown in the right panel of Fig. 2 . The prominent bump around $\operatorname{mag}_{\mathrm{K}}=15$ is typical for observations toward the GC and galactic bulge (Tiede, Frogel \& Terndrup 1995; Genzel, Schödel, Ott et al. 2003; Figer, Rich, Kim et al. 2004) and is due to horizontal branch/red clump (HB/RC) stars. These old stars of roughly $\geqslant 1 \mathrm{M}_{\odot}$ are the lightest ones in the examined magnitude range. Due to its age, this part of the stellar population may be relaxed by two-body encounters and bear signatures of mass segregation. In fact, as the right panel in Fig. 3 shows, the HB/RC stars display a flat or even slightly inverted slope at distances from $1-3^{\prime \prime}$ from Sgr $A^{*}$. Inside of $1^{\prime \prime}$, the cusp slope is very steep. Here, the stellar population in the chosen magnitude range consists almost entirely of B-type main sequence stars with masses around $10 \mathrm{M}_{\odot}$ (Eisenhauer, Genzel, Alexander et al. 2005). This is consistent with stellar dynamics that predicts a steeper power law index for the heavier components of the cluster (Bahcall \& Wolf 1977). As a caveat it must be said that it is not clear whether the young stars near Sgr A* had time to relax dynamically.

\section{References}

Schödel, R., Ott, T., Genzel, R. et al. 2003, ApJ, 596, 1015

Ghez, A. M., Duchêne, G., Matthews, K. et al. 2003, ApJ, 586, L127

Eisenhauer, F., Genzel, R., Alexander, T. et al. 2005, ApJ, 628, 246

Bahcall, J. N. \& Wolf, R. A. 1976, ApJ, 209, 214

Bahcall, J. N. \& Wolf, R. A. 1977, ApJ, 216, 883

Lightman, A. P. \& Shapiro, S. L. 1977, ApJ, 211, 244

Murphy, B. W., Cohn, H. N. \& Durisen, R. H. 1991, ApJ, 370, 60

Genzel, R., Schödel, R., Ott, T. et al. 2003, ApJ, 594, 812

Diolaiti, E., Bendinelli, O., Bonaccini, D. et al. 2000, A\&AS, 147, 48

Paumard, T., Genzel, R., Martins, F. et al. 2006, ApJ, 643, 1011

Draine, B. T. 1989, Proceedings of the 22nd Eslab Symposium held in Salamanca, Spain, 7-9 December, 1988, ed. B.H. Kaldeich, 93

Lutz, D., Krabbe, A. \& Genzel, R. 1993, ApJ, 418, 244

Melia, F., Coker, R. F. \& Yusef-Zadeh, F. 1996, ApJ, 460, L33

Yusef-Zadeh, F., Roberts, D. A. \& Biretta, J. 1998, ApJ, 499, L159

Schödel, R., Eckart, A., Iserlohe, C., Genzel, R. \& Ott, T. 2005 ApJ, 625, L111

Maillard, J. P., Paumard, T., Stolovy, S. R. \& Rigaut, V. 2004, A\&A, 423, 155

Figer, D., Rich, V., Kim, S.S., Morris, M. \& Serabyn, E. 2004, ApJ, 601, 319

Tiede, G. P., Frogel, J. A. \& Terndrup, D. M. 1995, AJ, 110, 2788

EMmanuil VilkoviskiY: What is the total mass of the cusp and the stellar cluster?

RAINER SchÖDEL: The cusp has mass of about $10^{3} M_{\odot}$.

YIPING WANG: Do you see any metallicity gradient towards the central part of the Galactic center?

RAINER SCHÖDEL: No, we don't have enough information on the metallicity of the central star cluster, however, it is my understanding that solar metallicity is entirely consistent with available data. 